\title{
An English Network Teaching Method Supported by Artificial Intelligence Technology and WBIETS System
}

\author{
Haibao Du \\ Teaching Section of Foreign Languages, Shenyang Normal University, Shenyang, China \\ Correspondence should be addressed to Haibao Du; harlindu@synu.edu.cn
}

Received 27 May 2021; Revised 25 June 2021; Accepted 7 August 2021; Published 29 August 2021

Academic Editor: Mian Ahmad Jan

Copyright (C) 2021 Haibao Du. This is an open access article distributed under the Creative Commons Attribution License, which permits unrestricted use, distribution, and reproduction in any medium, provided the original work is properly cited.

\begin{abstract}
The traditional English teaching system has certain problems in the acquisition of teaching resources and the innovation of teaching models. In order to improve the effect of subsequent English online teaching, this paper improves the machine learning algorithm to make it a core algorithm that can be used by artificial intelligence systems. Moreover, this paper combines the WBIETS system to expand the system function, analyzes the needs of the English network teaching system, and constructs the system function modules and logical structure. The data layer, logic layer, and presentation layer in the system constructed in this paper are independent of each other and can be effectively expanded when subsequent requirements change. In addition, this paper solves the problem of acquiring English teaching resources through the WBIETS system. To evaluate the performance of the English network teaching system, this paper performs comprehensive mathematical and experimental analysis. The experimental results show that the system constructed in this paper basically meets the actual teaching requirements.
\end{abstract}

\section{Introduction}

English is compulsory for education around the globe and a mandatory course in most universities. In the past two years, due to COVID-19, most of the on-site English teaching has transformed to online teaching, but traditional online teaching faces certain challenges that hinder its widespread adoption. Based on these challenges and shortcomings, this paper analyzes the traditional English network teaching system and combines artificial intelligence technology and the WBIETS system to construct an English network teaching system. With the deepening of online education, educational sponsors and students have reflected more profound problems [1]. After starting the course, the problem of how to quickly and accurately find the required resources has become increasingly prominent that has raised new questions for the development of Internet education platforms [2].

The online education system is a system platform established to express educational content and implement educational activities through the Internet. On the Internet teaching platform, teachers can answer questions online.
These functions make a great contribution to traditional educational activities. However, with the long-term operation of the system, the unreasonable structure of the website has been exposed. Moreover, in the process of students' selflearning, there are problems such as being unable to face a large amount of information [3]. In the use of the system, many user access logs are stored on the website. The major contributions of this work are as follows:

(1) Through the WBETS mining analysis of these log records, the user's access patterns are discovered and analyzed.

(2) Moreover, useful user action patterns are applied to website structure adjustment and page push services. When users with similar characteristics visit the website again, they are pushed to the relevant network resources to realize the personalized and humanized services of the website.

The rest of this paper is organized as follows. In Section 2 , the related works from the literature are provided. In Section 3, the algorithmic analysis of the English network teaching system is discussed. In Section 4, the construction 
and performance analysis of the English network teaching system based on artificial intelligence and the WBIETS system are elaborated. Finally, we conclude the paper and future research directions are provided in Section 5.

\section{Related Works}

For technical research and development of a smart education system, the researchers used the open source software of smart education to develop their own new functions based on the existing design. The design of online course resources involves teachers integrating their own courses into the platform of wisdom education, increasing the educational resources of the teaching plan and various educational activities, and applying them to actual teaching to allow teachers to experience guidance [4]. Educational design based on wisdom education involves various links and factors in education, such as analyzing the educational objectives of the curriculum, the weight of the curriculum, students, learning content, educational methods, learning activity design, learning evaluation, and other links [5].

Wisdom educational knowledge management has functions and characteristics, which can realize personal knowledge management and play an important role in education. Through the smart education system, teachers organize the knowledge and experience of education courses into a network platform and provide them to learners. After that, learners can manage their own knowledge while learning it [6]. Online education provides learners with an educational platform through the intelligent education system to improve their learning ability. Moreover, it uses the online learning of the intelligent education system to train teachers and students. The system evaluation of smart education includes the comparison of the advantages and disadvantages of the smart education system and other online education systems, its functional characteristics and the evaluation of each module, and complete recommendations [7]. At present, smart education mostly focuses on theoretical research and evaluation but lacks the analysis of data in the education system and the applied research that actually enters education. In addition, in higher vocational colleges, the application of intelligent education network education system is less popular, and educational resources need to be developed and integrated, and there is a lack of many excellent demonstration courses [8].

In [9], it was proposed that the structured system should be free to choose the content which is of interest to learners and then provide suggestions and guidance to students according to the actual situation. The system designed in [10] has functional modules related to the evaluation of learners' behavior. The system design proposed in [11] is mainly composed of learners' discovery of learning goals, learning behaviors, and personalized learning. In [12], the authors analyzed learners for individual learning and promoted the transmission of learning resources. In [13], the authors developed a course signal project to judge the current academic performance of students through the student information system, content management system, and student scores.
From the above analysis, the current research of experts and scholars on online teaching is mostly focused on pushing resources and the reproduction of traditional teaching models in online teaching, which is not good for modern English teaching. To address the aforementioned shortcoming, this paper uses artificial intelligence algorithms to create an innovative construction of English network teaching system.

\section{Algorithmic Analysis of English Network Teaching System}

The number of layers of deep neural network (DN) determines the ability to fit complex functions. The DN uses backpropagation to solve the problem of local optimal solution of optimization function. However, the upper neuron in the DN structure can form connections, the signal direction of neuron propagation is fixed, each time point is relatively independent, and it cannot work according to changes in time series. The chronological order of the text sequence is very important for natural language processing applications [14].

This paper improves on the extraction of recurrent neural network (RNN). In addition to the output of the $(n-1)$ layer of neurons at time $t$, it also includes its own output at time $(m-1)$. This article also incorporates the student emotion recognition model in the algorithm, as shown in Figure 1.

As shown in Figure 2, $X_{t-1}$ is input of the input layer at time $t-1$, and the hidden layer has an initialization state, that is, the initial value $h_{0}$. The $h_{t-1}$ unit in the hidden layer will perform weight calculations, and $X_{t-1}$ and $h_{0}$ will perform hyperbolic tangent function calculations using the following equation [15]:

$$
\begin{aligned}
& h_{t-1}=\tanh \left(W\left[h_{0}, x_{t-1}\right]+b\right), \\
& f(x)=\tanh (x)=\frac{\sinh (x)}{\cosh (x)}=\frac{e^{x}-e^{-x}}{e^{x}+e^{-x}}
\end{aligned}
$$

If the hidden layer's weight parameter is different from the input layer's weight parameter, then

$$
h_{t}=f\left(\left[U\left(h_{t-1}\right)+W\left(x_{t}\right)\right]\right) \text {. }
$$

Through calculation, the hidden layer state $h_{t-1}$ is generated, and the result $O_{t-1}$ is output at the same time. Among them, $h_{t-1}$ cannot be output directly and needs to be normalized using the softmax function. Before softmax processing, $h_{t-1}$ needs to be multiplied by a weight matrix $V$, using the following equation[16]:

$$
\begin{aligned}
h_{t} & =\tanh \left(W\left[h_{t-1}, y_{t}, C\right]+b\right), \\
O_{t-1} & =\operatorname{soft} \max \left(V h_{t-1}+c\right) .
\end{aligned}
$$

In the same way, the hidden layer state $h_{t-1}$ generated at time $t-1$ and the input $X_{t}$ at time $t$ are used as the input of the $h_{t}$ neuron and the hyperbolic tangent operation is performed to generate the hidden layer $h_{t}$ state. At the same time, softmax is used for normalization to output the result $O_{t}$. 


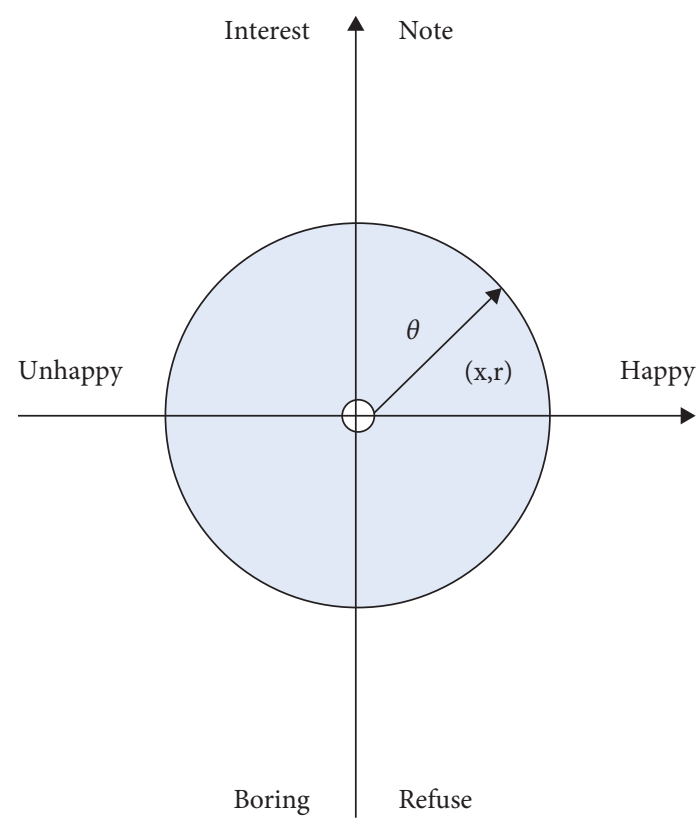

FIGURE 1: Student emotion model.

When solving the actual text processing problem, most of the input and output sequences are not the same length, so in order to meet the particularity of natural language processing, an $N-M$ transform is performed on the basis of the RNN network, that is, the encoder-decoder model. The whole model is divided into two parts: encoder and decoder. The encoder converts a changeable input original text sequence into a fixedlength meaning vector $C$. The decoder converts the meaning vector $C$ with a fixed length into a target text sequence $S=$ $\left\{W_{1}^{S}, W_{2}^{S}, \ldots, W_{n}^{S}\right\}$ with a variable length [17].

In this paper, RNN is described as the basic network structure of the encoder layer and decoder layer. The main task of the encoder layer is to process the input layer data and compress the input sequence into a vector of specified length. This vector is called the meaning vector $C$. The decoder layer is mainly used to generate output, generate a specified sequence based on the meaning vector, and use the meaning code $C$ to connect between encoder and decoder. There are two ways to use meaning vectors in the decoder.

(1) The semantic vector $C$ in the coding layer is only used as an initial state to participate in the operation, and all operations after the decoding layer have nothing to do with the semantic vector $C$. In other words, the meaning vector only works at the beginning.

(2) Another processing method is that the word sense vector $C$ participates in the calculation of the sequence at all times, and different vectors that generate the semantic vector at different times work on the decoder.

As shown in Figure 3, the encoder first performs RNN processing on the input sentence. Moreover, unlike the steps that are not output, the calculation result of each hidden layer neural unit directly serves as the input of the next neuron. After that, through the RNN, the intermediate meaning vector $C$ is obtained [18].

As shown in Figure 3, the encoder first performs RNN processing on the input sentence. The difference is that there is no output in this step, but the calculation result of each hidden layer neural unit is directly used as the input of the next neuron. Then, the intermediate semantic vector $C$ is obtained through the RNN, in which the final state $h_{4}$ can be obtained or $h_{4}$ can be subjected to functional operations. The function formula is [19]

$$
C=\tanh \left(U h_{t}\right),
$$

where $U$ is the weight matrix and $h_{t}$ is the last hidden layer state of the encoder layer. The initial value $h_{0}$ and the start signal $y_{0}$ are operated together with the semantic vector $C$ to generate the hidden layer state $h_{1}$ and generate $y_{1}$. Then, $h_{1}, y_{1}$, and the semantic vector $C$ are budgeted together to generate the hidden layer state $h_{2}$ and output $y_{2}$, which are passed on in turn, and the final output result is obtained. The formula is

$$
\begin{aligned}
h_{t} & =\tanh \left(W\left[h_{t-1}, y_{t}, C\right]+b\right), \\
O_{t} & =\operatorname{soft} \max \left(V h_{t}+c\right) .
\end{aligned}
$$

The meaning of $C$ as a semantic vector is to play a role in every moment of its decoder. It can be expressed as

$$
\begin{aligned}
h_{1} & =\tanh \left(W\left[h_{0}, y_{0}, C\right]+b\right), \\
O_{1} & =\operatorname{soft} \max \left(V h_{1}+c\right), \\
h_{2} & =\tanh \left(W\left[h_{1}, y_{1}, C\right]+b\right), \\
O_{2} & =\operatorname{soft} \max \left(V h_{2}+c\right), \\
h_{2} & =\tanh \left(W\left[h_{1}, y_{1}, C\right]+b\right), \\
O_{t} & =\operatorname{soft} \max \left(V h_{n}+c\right) .
\end{aligned}
$$

$O_{t}$ is the output vector at each time, the vector dimension is the length of the vocabulary, and each value of the vector is the probability corresponding to the term. When the probability of the predicted value end signal is the greatest, the prediction ends. The application of the encoder-decoder principle solves the problem of the input and output of the text sequence of the training model. Encoder processes the input sequence, the intermediate meaning vector $C$ stores sequence information, and decoder processes the output sequence, thus realizing the processing from beginning to end.

In the natural language generation task, the LSTM neural network, which is a variant of the recurrent neural network, is usually used as the basic network of the decoder in the architecture. Long short-term memory (LSTM) is a time loop neural network that solves the gradient dispersion problem of traditional RNN models. Now, the LSTM network model is used for generating operations with text sequences [20]. In this model, the pagoda memory model is incorporated, as shown in Figure 4.

$\sigma$ represents that the control unit controls input, output, and storage, respectively. The control unit is composed of a sigmoid function and a point power action. The value of the sigmoid function is between 0 and 1 , and the transmission of information is determined by the 


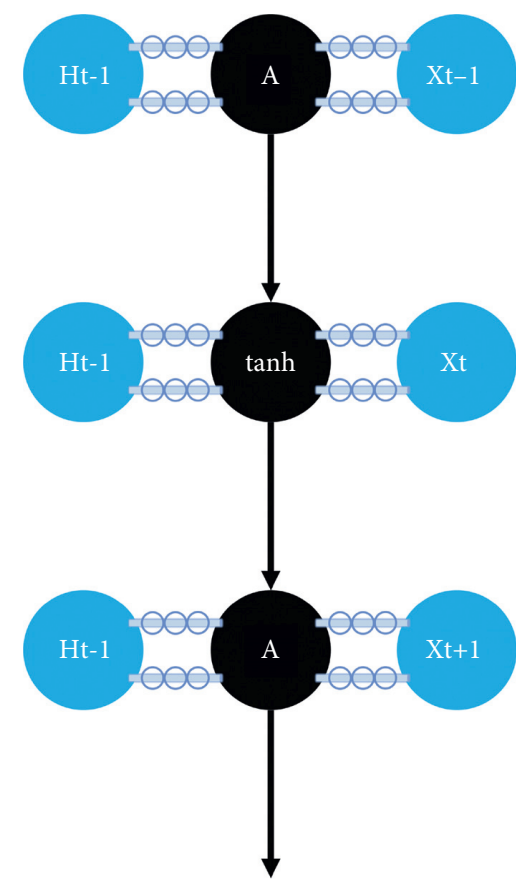

FIgURE 2: Network computing layer.

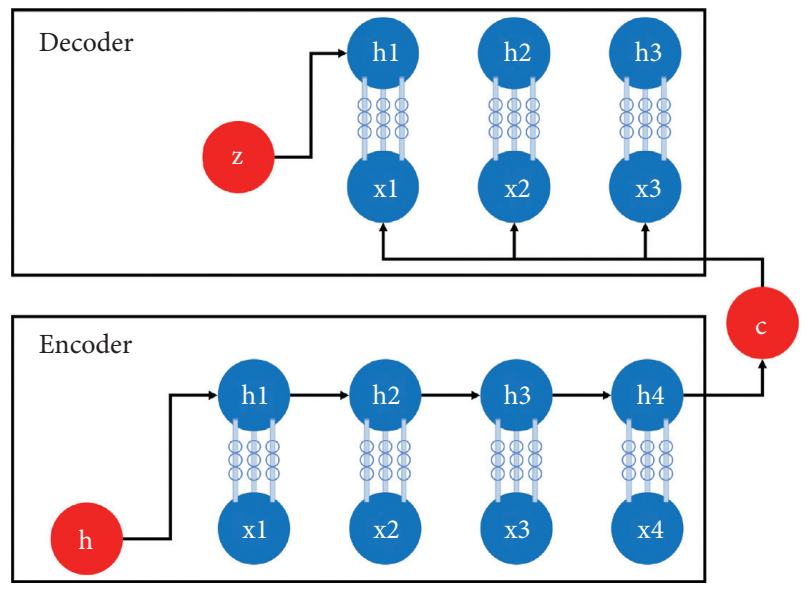

Figure 3: Encoder-decoder structure.

point multiplication operation. At 0 , the information is not transmitted, and at 1 , the information is all forwarded. The model also joins the cell-state conveyor belt, which is responsible for the storage space of the entire model, and the control unit controls the storage of the storage unit during time conversion. The storage department is responsible for deleting the information required by the three control departments [21].

The input unit stores some current information and combines the past and current storage into the following formula:

$$
\begin{aligned}
i_{t} & =\sigma\left(W_{i} \cdot\left[h_{t-1}, x_{t}\right]+b_{i}\right), \\
\widetilde{C}_{t} & =\tanh \left(w_{c} \cdot\left[h_{t-1}, x_{t}\right]+b_{c}\right), \\
\widetilde{C}_{t} & =f_{t} * C_{t-1}+i_{t} * \widetilde{C}_{t} .
\end{aligned}
$$
follows:

The output unit is responsible for the final output as

$$
\begin{aligned}
O_{t} & =\sigma\left(W_{o} \cdot\left[h_{t-1}, x_{t}\right]+b_{o}\right), \\
h_{t} & =O_{t} * \tanh \left(C_{t}\right) .
\end{aligned}
$$

By combining the BERT model with the LSTM neural network, text can be automatically generated. In the encoder part, the BERT model can be selected as the encoder in our frame, and the BERT can complete the task of extracting the complex feature information contained in the input text as a bidirectional transformer encoder in multiple layers. This two-way encoding feature solves the word ambiguity problem that is difficult to solve by the traditional model and ensures that the words in each position can pay attention to the information of the adjacent position words. At the same time, the network depth of the 12-layer BERT model can present different characteristic information of each layer through deep analysis of the text.

We use a two-layer LSTM neural network as part of the decoder. In the transformer network structure, the input is mainly composed of word coding and position coding. Position coding is mainly used to record the position information in the input sequence of each term [22].

\section{Construction of English Network Teaching System Based on Artificial Intelligence and WBIETS}

Aiming at the current situation of English online teaching, this paper analyzes the needs of the teaching system and analyzes the actual situation of the system. The system constructed in this paper needs to have system management function, which is the intelligent system of all network teaching systems. Also, the system needs to have a teaching function, which is also the main function of this system. Finally, the system needs to have the function of students' autonomous learning, so the system role setting in this paper mainly includes system administrators, teachers, and students. The corresponding functional management modules mainly include system management, teaching management, and learning management, corresponding to three roles, respectively. The English network teaching system obtained on this basis is shown in Figure 5.

The system server in this paper stores all the information in the system's self-built database, which is convenient for unified management and modification of information. The data layer, logic layer, and presentation layer in the system constructed in this paper are all 


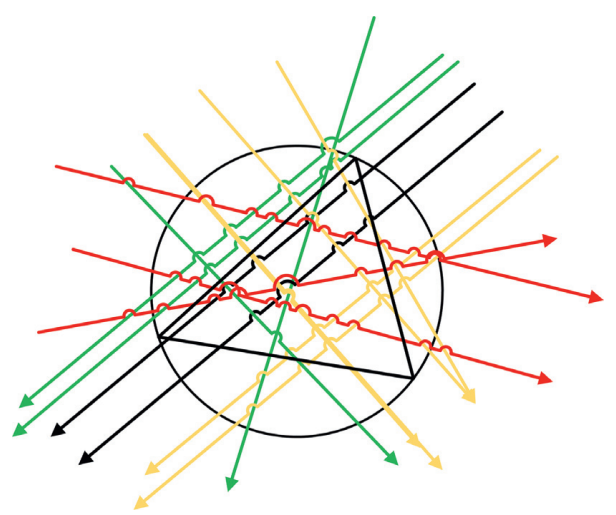

Figure 4: Pagoda memory model.

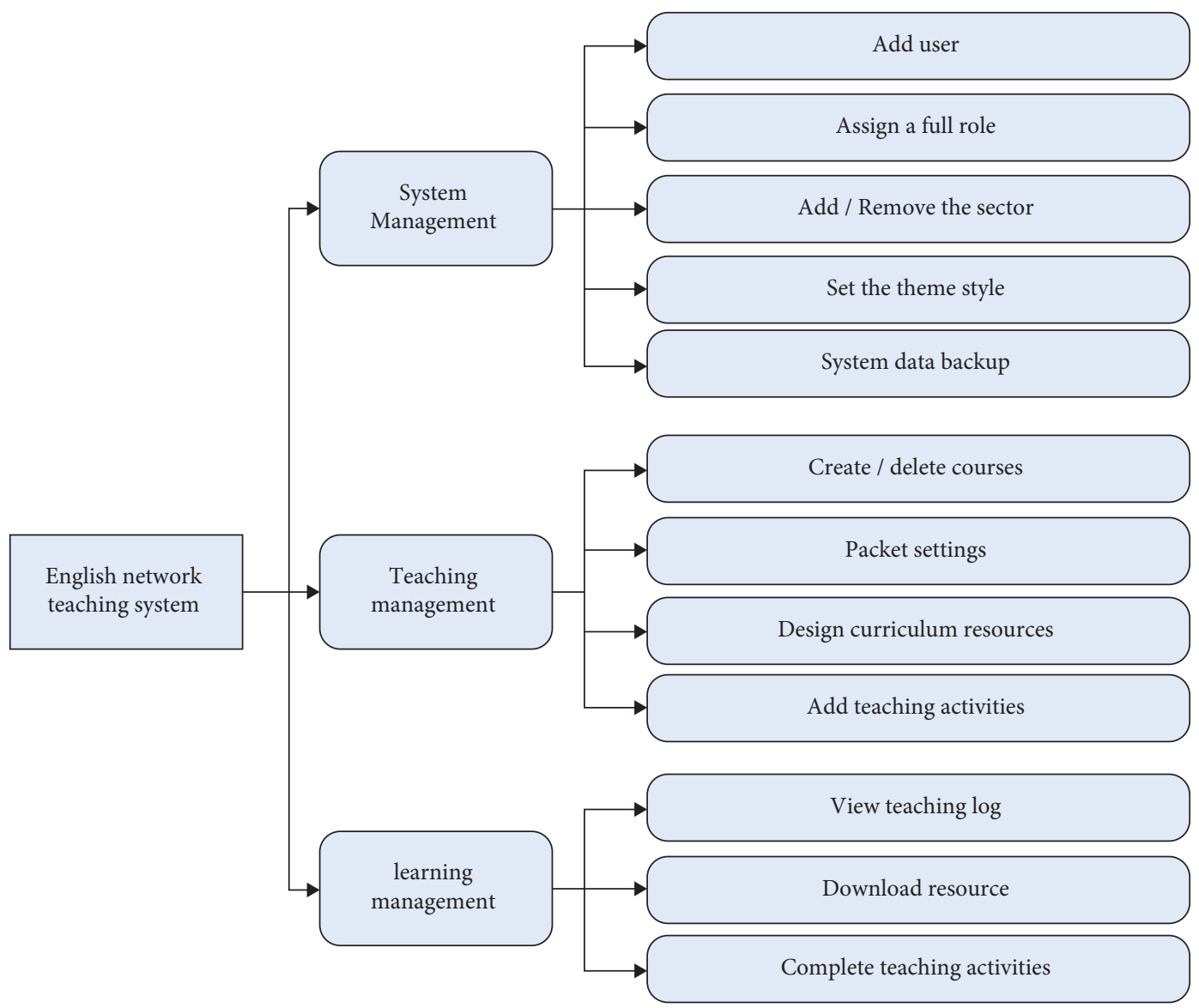

FIGURE 5: Structure diagram of English network teaching system.

independent of each other, so the system can be effectively expanded when subsequent requirements change. The system constructed in this paper is mainly carried out through the WBIETS system during data processing. The system can provide administrators, teachers, and students with relevant ways to operate the system. Based on the above analysis, the relationship between the elements of the system constructed in this paper can be expressed as the form shown in Figure 6.
From the above analysis, the construction of the English network teaching system is completed. Moreover, the effect of the English teaching system can be verified by system simulation through the simulation system. After that, this paper analyzes the English network teaching system constructed in this paper. The innovation of this system lies in the collection and processing of network data through WBIETS, which enables the teaching system constructed in this paper to be used as English teaching 


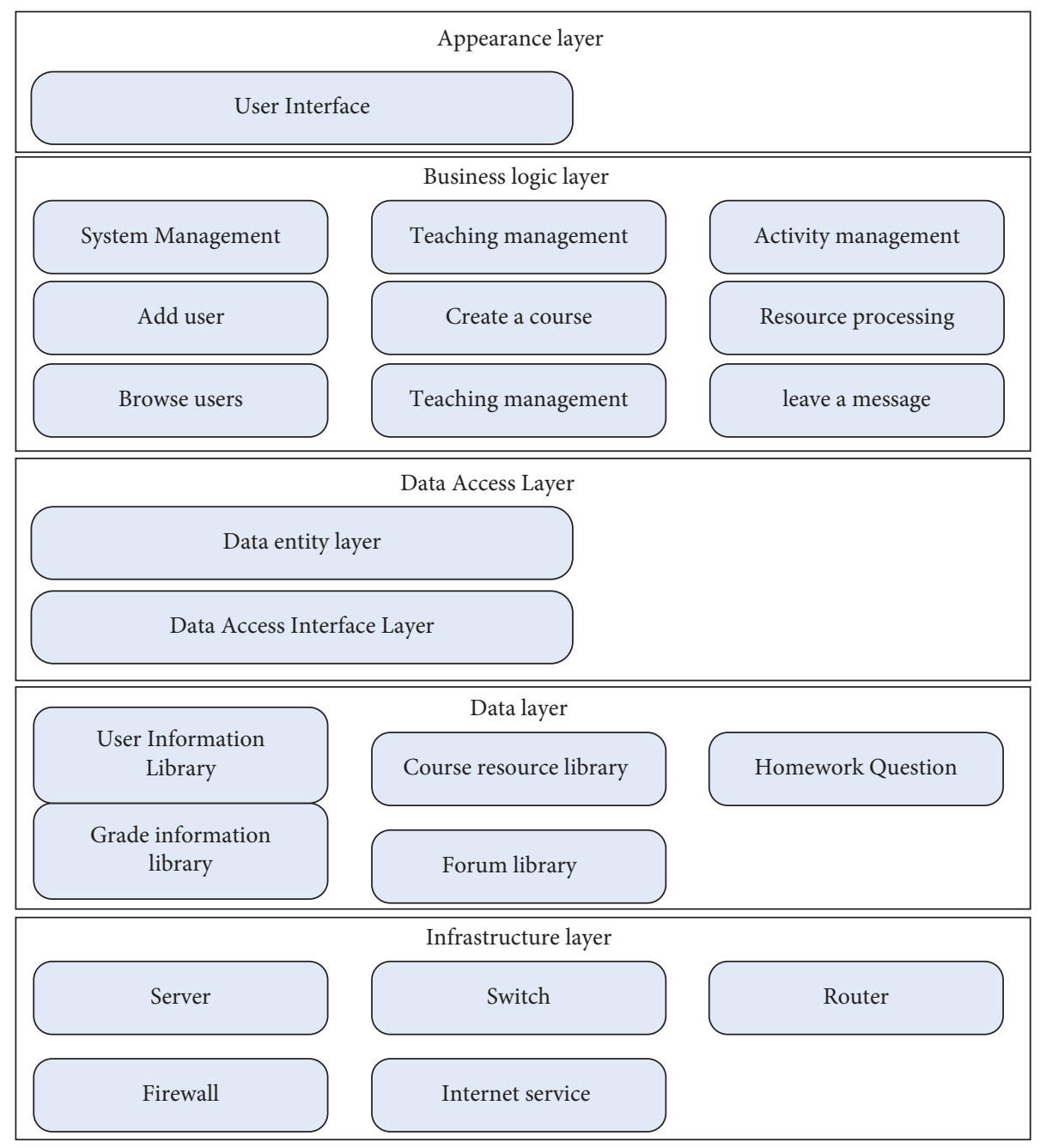

FIGURE 6: System function structure diagram.

resources. Therefore, in the system evaluation, this paper mainly researches the system data collection and data processing effect and teaching effect. Among them, data collection and data processing can be directly referred to as the acquisition and processing of teaching resources. In the experimental research, this paper first evaluates the acquisition and processing effects of the WBIETS system on teaching resources and obtains the corresponding data through simulation. The statistical data are shown in Table 1 and Figure 7.

Through the statistics of the acquisition and processing effect of WBIETS teaching resources, it can be known that the system constructed in this paper has certain advantages compared with the traditional model in the acquisition of English teaching resources. On this basis, this paper evaluates the teaching effect of the system through the teaching experiment scoring method, and the results obtained are shown in Table 2 and Figure 8.

From the above experimental analysis results, the system constructed in this paper has a good English teaching system, which can play a certain effect in modern English teaching, and subsequent experimental teaching can be carried out through the system constructed in this paper. 
TABLE 1: The effect of acquisition and processing of WBIETS system on teaching resources.

\begin{tabular}{lccccc}
\hline Num & Resource collection & Resource handling & Num & Resource collection & Resource handling \\
\hline 1 & 91.9 & 82.8 & 21 & 94.3 & 76.2 \\
2 & 94.6 & 87.9 & 22 & 93.7 & 80.4 \\
3 & 90.7 & 86.4 & 23 & 91.6 & 83.6 \\
4 & 94.4 & 84.3 & 24 & 94.7 & 87.8 \\
5 & 92.2 & 78.8 & 25 & 91.8 & 82.7 \\
6 & 90.5 & 76.3 & 26 & 92.2 & 85.6 \\
7 & 92.2 & 76.4 & 27 & 90.6 & 82.9 \\
8 & 93.5 & 79.5 & 28 & 90.7 & 87.4 \\
9 & 91.4 & 76.3 & 29 & 94.2 & 87.8 \\
10 & 92.7 & 37.0 & 31 & 92.4 & 83.4 \\
11 & 93.1 & 87.9 & 32 & 93.9 & 83.9 \\
12 & 92.0 & 87.6 & 33 & 94.4 & 75.6 \\
13 & 90.9 & 75.5 & 34 & 92.6 & 83.2 \\
14 & 91.5 & 78.8 & 35 & 92.7 & 76.9 \\
15 & 93.6 & 84.6 & 36 & 91.9 & 75.2 \\
17 & 92.0 & 78.8 & 37 & 92.0 & 83.7 \\
18 & 93.5 & 80.7 & 38 & 93.1 & 79.3 \\
20 & 94.9 & 75.1 & 39 & 91.2 & \\
\hline
\end{tabular}

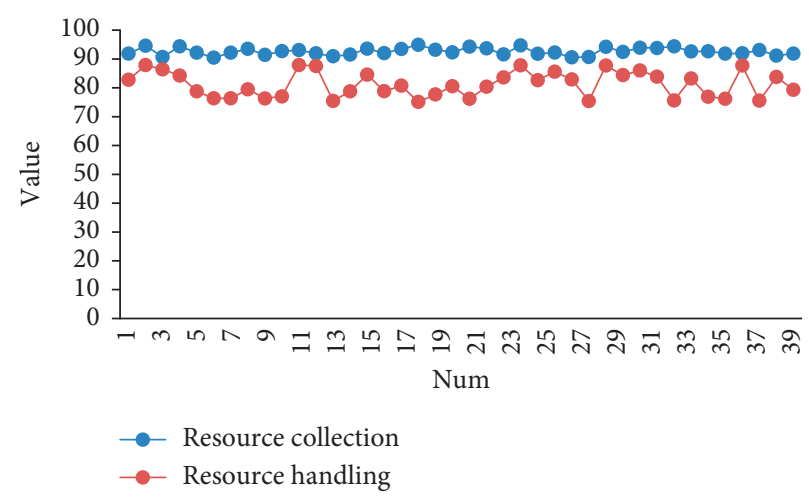

FIGURE 7: Effect of acquisition and processing of WBIETS system on teaching resources.

TABLE 2: Evaluation of system teaching effect.

\begin{tabular}{|c|c|c|c|c|c|c|c|}
\hline Num & Teaching effect & Num & Teaching effect & Num & Teaching effect & Num & Teaching effect \\
\hline 1 & 73.5 & 21 & 92.0 & 41 & 74.2 & 61 & 83.8 \\
\hline 2 & 82.8 & 22 & 95.4 & 42 & 85.8 & 62 & 91.7 \\
\hline 3 & 91.8 & 23 & 71.6 & 43 & 75.2 & 63 & 84.9 \\
\hline 4 & 88.1 & 24 & 86.2 & 44 & 76.3 & 64 & 88.1 \\
\hline 5 & 76.7 & 25 & 81.0 & 45 & 80.9 & 65 & 77.0 \\
\hline 6 & 82.1 & 26 & 73.8 & 46 & 82.5 & 66 & 83.2 \\
\hline 7 & 84.9 & 27 & 86.6 & 47 & 83.1 & 67 & 93.6 \\
\hline 8 & 91.0 & 28 & 88.0 & 48 & 84.7 & 68 & 85.6 \\
\hline 9 & 90.9 & 29 & 77.2 & 49 & 84.2 & 69 & 75.1 \\
\hline 10 & 71.7 & 30 & 83.0 & 50 & 77.3 & 70 & 81.2 \\
\hline 11 & 75.5 & 31 & 71.7 & 51 & 89.6 & 71 & 74.2 \\
\hline 12 & 78.8 & 32 & 81.7 & 52 & 79.0 & 72 & 86.5 \\
\hline 13 & 89.2 & 33 & 76.6 & 53 & 78.8 & 73 & 72.6 \\
\hline 14 & 92.5 & 34 & 83.7 & 54 & 73.2 & 74 & 90.6 \\
\hline 15 & 89.9 & 35 & 93.5 & 55 & 81.3 & 75 & 87.1 \\
\hline 16 & 72.4 & 36 & 93.3 & 56 & 85.0 & 76 & 76.4 \\
\hline 17 & 71.9 & 37 & 87.2 & 57 & 88.8 & 77 & 81.4 \\
\hline 18 & 93.6 & 38 & 83.8 & 58 & 90.0 & 78 & 75.6 \\
\hline 19 & 90.7 & 39 & 79.5 & 59 & 88.6 & 79 & 84.1 \\
\hline 20 & 88.1 & 40 & 94.4 & 60 & 77.9 & 80 & 85.4 \\
\hline
\end{tabular}




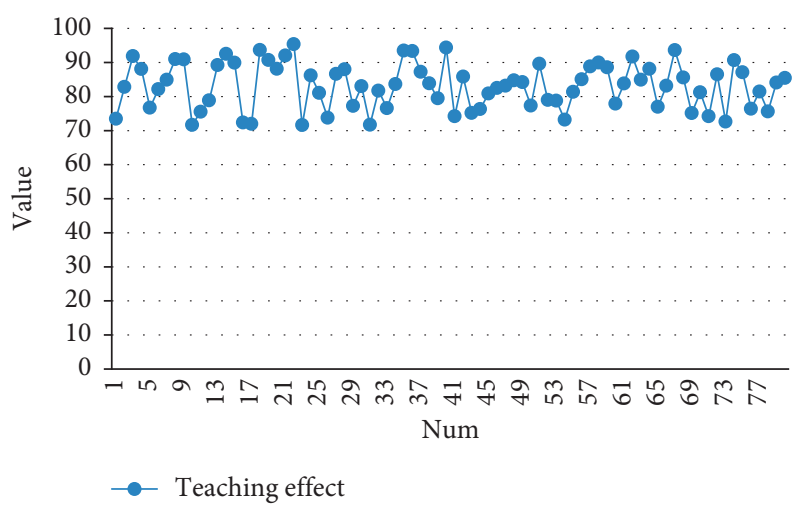

Figure 8: Evaluation of system teaching effect.

\section{Conclusion}

In order to study the system that can be used for English network intelligent teaching, this paper combines artificial intelligence and the WBIETS system to construct an English network teaching system. The data layer, logic layer, and presentation layer in the system constructed in this paper are all independent of each other, so the system can be effectively expanded when subsequent requirements change. Moreover, the data processing of the system constructed in this paper is mainly carried out through the WBIETS system, which can provide administrators, teachers, and students with relevant ways of system operation. In addition, this paper uses an improved algorithm as a base for system design by expanding and constructing it based on the original WBIETS system. In this way, the logical structure and functional modules of the system are obtained to configure the system's response users. Finally, this paper conducts system performance analysis through experimental analysis. From the experimental results, it can be seen that the English network teaching system constructed in this paper can meet the expected teaching needs of an organization.

\section{Data Availability}

The data that support the findings of this study are available from the corresponding author upon reasonable request.

\section{Conflicts of Interest}

The author declares that there are no conflicts of interest concerning this work.

\section{References}

[1] S. Amershi, M. Cakmak, W. B. Knox, and T. Kulesza, "Power to the people: the role of humans in interactive machine learning," AI Magazine, vol. 35, no. 4, pp. 105-120, 2014.

[2] S. Basith, B. Manavalan, T. H. Shin, and G. Lee, "SDM6A: a web-based integrative machine-learning framework for predicting 6ma sites in the rice genome," Molecular Therapy-, vol. 18, no. 6, pp. 131-141, 2019.

[3] D. Bzdok and A. Meyer-Lindenberg, "Machine learning for precision psychiatry: opportunities and challenges," Biological
Psychiatry: Cognitive Neuroscience and Neuroimaging, vol. 3, no. 3, pp. 223-230, 2018.

[4] J. Cai, J. Luo, S. Wang, and S. Yang, "Feature selection in machine learning: a new perspective," Neurocomputing, vol. 300, no. 2, pp. 70-79, 2018.

[5] M. Chen, Y. Hao, K. Hwang, L. Wang, and L. Wang, "Disease prediction by machine learning over big data from healthcare communities," Ieee Access, vol. 5, no. 1, pp. 8869-8879, 2017.

[6] A. Chowdhury, E. Kautz, B. Yener, and D. Lewis, "Image driven machine learning methods for microstructure recognition," Computational Materials Science, vol. 123, no. 8, pp. 176-187, 2016.

[7] C. W. Coley, R. Barzilay, T. S. Jaakkola, W. H. Green, and K. F. Jensen, "Prediction of organic reaction outcomes using machine learning," ACS Central Science, vol. 3, no. 5, pp. 434-443, 2017.

[8] H. Darabi, B. Choubin, O. Rahmati, A. Torabi Haghighi, B. Pradhan, and B. Kløve, "Urban flood risk mapping using the GARP and QUEST models: a comparative study of machine learning techniques," Journal of Hydrology (Amsterdam), vol. 569, no. 5, pp. 142-154, 2019.

[9] P. Feng, B. Wang, D. L. Liu, C. Waters, and Q. Yu, "Incorporating machine learning with biophysical model can improve the evaluation of climate extremes impacts on wheat yield in south-eastern Australia," Agricultural and Forest Meteorology, vol. 275, no. 3, pp. 100-113, 2019.

[10] C. Folberth, A. Baklanov, J. Balkovič, R. Skalský, N. Khabarov, and M. Obersteiner, "Spatio-temporal downscaling of gridded crop model yield estimates based on machine learning," Agricultural and Forest Meteorology, vol. 264, no. 4, pp. 1-15, 2019.

[11] J. N. Goetz, A. Brenning, H. Petschko, and P. Leopold, "Evaluating machine learning and statistical prediction techniques for landslide susceptibility modeling," Computers \& Geosciences, vol. 81, no. 1, pp. 1-11, 2015.

[12] L. Itu, S. Rapaka, T. Passerini et al., "A machine-learning approach for computation of fractional flow reserve from coronary computed tomography," Journal of Applied Physiology, vol. 121, no. 1, pp. 42-52, 2016.

[13] K. Kourou, T. P. Exarchos, K. P. Exarchos, M. V. Karamouzis, and D. I. Fotiadis, "Machine learning applications in cancer prognosis and prediction," Computational and Structural Biotechnology Journal, vol. 13, no. 5, pp. 8-17, 2015.

[14] F. A. Narudin, A. Feizollah, N. B. Anuar, and A. Gani, "Evaluation of machine learning classifiers for mobile malware detection," Soft Computing, vol. 20, no. 1, pp. 343-357, 2016. 
[15] A. Rajkomar, J. Dean, and I. Kohane, "Machine learning in medicine," New England Journal of Medicine, vol. 380, no. 14, pp. 1347-1358, 2019.

[16] V. Rodriguez-Galiano, M. Sanchez-Castillo, M. Chica-Olmo, and M. Chica-Rivas, "Machine learning predictive models for mineral prospectivity: an evaluation of neural networks, random forest, regression trees and support vector machines," Ore Geology Reviews, vol. 71, no. 3, pp. 804-818, 2015.

[17] J. Sieg, F. Flachsenberg, and M. Rarey, "In In need of bias control: evaluating chemical data for machine learning in structure-based virtual screening," Journal of Chemical Information and Modeling, vol. 59, no. 3, pp. 947-961, 2019.

[18] F. Thabtah and D. Peebles, "A new machine learning model based on induction of rules for autism detection," Health Informatics Journal, vol. 26, no. 1, pp. 264-286, 2020.

[19] C. Voyant, G. Notton, S. Kalogirou et al., "Machine learning methods for solar radiation forecasting: a review," Renewable Energy, vol. 105, no. 2, pp. 569-582, 2017.

[20] L. Ward, A. Agrawal, A. Choudhary, and C. Wolverton, "A general-purpose machine learning framework for predicting properties of inorganic materials," npj Computational Materials, vol. 2, no. 1, pp. 1-7, 2016.

[21] Y. Xin, L. Kong, Z. Liu et al., "Machine learning and deep learning methods for c," IEEE Access, vol. 6, no. 1, pp. 35365-35381, 2018.

[22] Q. Yao, H. Yang, R. Zhu et al., "Core, mode, and spectrum assignment based on machine learning in space division multiplexing elastic optical networks," IEEE Access, vol. 6, no. 6, pp. 15898-15907, 2018. 